\title{
Rydberg States of the Helium Atom
}

\author{
JIA DENG, ANDREW T. B. GILBERT, PETER M. W. GILL \\ Research School of Chemistry, Australian National University, Canberra ACT 0200, Australia
}

Received 28 October 2008; accepted 18 November 2008

Published online 6 March 2009 in Wiley InterScience (www.interscience.wiley.com).

DOI 10.1002/qua.22015

\begin{abstract}
Using the recently introduced maximum overlap method and Hartree-Fock Perturbation Theory (HFPT), we compute Hartree-Fock (HF) wavefunctions for triplet $1 \mathrm{~s} n \mathrm{~s}$ states of the helium atom. Comparison with near-exact results from Nakatsuji's free ICI method reveals that HF theory provides a simple route to accurate energies of these Rydberg states, especially for large $n$. (C) 2009 Wiley Periodicals, Inc. Int J Quantum Chem 109: 1915-1919, 2009
\end{abstract}

Key words: Rydberg states; excited states; helium; maximum overlap method; Hartree-Fock restoration

\section{Introduction}

D uring an illustrious career, Hirao has made many contributions to the discipline of quantum chemistry. One of his recent contributions concerns the development of systematic methods for improving the poor treatment of Rydberg and charge-transfer excitations afforded by timedependent density functional theory (TD-DFT) with traditional functionals. Several years ago, he and his coworkers introduced [1] long-range-corrected density functional theory (LC-DFT) in which the Ewald [2-5] or erfgau [6, 7] partition is used to separate the Coulomb operator into a short-range part that is treated by density functional theory [8] and a long-range part that is treated using conventional wavefunction approaches [9]. As a result of Hirao's work, which has subsequently been adopted and adapted by several other groups, it is now possible

Correspondence to: P. M. W. Gill; e-mail: peter.gill@anu.edu.au to use TD-DFT to model both Rydberg and chargetransfer excitations with an accuracy comparable with that of valence excitations.

While Hirao was developing LC-DFT, Nakatsuji's group was introducing and exploring two impressive new approaches-the ICI method [10] and the free ICI method [11] — that yield near-exact solutions to the Schrödinger equation for small atomic and molecular systems. Recently, they have applied their approach to compute the ground-state energy of the helium atom to an astonishing 43 decimal digits [12] and also to obtain spectacularly accurate energies [13] for its singlet and triplet $1 s n s$ states, for $n=2,3, \ldots, 24$.

In those states where $n \gg 1$, the electrons are spatially well separated and one might anticipate intuitively that they will be weakly correlated and that the Hartree-Fock (HF) method, which neglects such effects, may be an excellent approximation. Until recently, it has been difficult to test such predictions because traditional self-consistent field (SCF) algorithms struggle to locate highly excited solutions, tending instead to collapse to the lowest SCF 
solution with the same spin and spatial symmetry as the initial guess.

However, with the advent of the maximum overlap method (MOM) [14-16] and Hartree-Fock Perturbation Theory [17] for finding and refining excited-state solutions to SCF equations, one can now examine this prediction with relative ease. In the remainder of this article, we therefore investigate the accuracy with which the conceptually and computationally straightforward HF method approximates the exact energies of the $1 s n s$ states of the helium atom, confining our attention to the triplet states to avoid the fundamental difficulties associated with the single-determinant description of open-shell singlets. Atomic units are used throughout.

\section{Zeroth-order approximation}

Before examining the performance of HF theory, it is illuminating to consider an even simpler independent particle model. In a $1 s n s$ state where $n \gg 1$, the nuclear shielding by the inner electron is almost perfect and one can imagine electrons 1 and 2 occupying the $\mathrm{He}^{+} 1 s$ orbital and an $\mathrm{H} n s$ orbital, respectively.

This physical picture corresponds to partitioning the full Hamiltonian

$$
H=-\frac{\nabla_{1}^{2}}{2}-\frac{\nabla_{2}^{2}}{2}-\frac{2}{r_{1}}-\frac{2}{r_{2}}+\frac{1}{r_{12}}
$$

into a nonsymmetric, noninteracting zeroth-order part

$$
H^{(0)}=\left(-\frac{\nabla_{1}^{2}}{2}-\frac{2}{r_{1}}\right)+\left(-\frac{\nabla_{2}^{2}}{2}-\frac{1}{r_{2}}\right)
$$

and a perturbative correction

$$
V=\frac{1}{r_{12}}-\frac{1}{r_{2}}
$$

$H^{(0)}$ is a sum of two hydrogenic Hamiltonians and standard solutions yield

$$
\begin{gathered}
\Psi_{n}^{(0)}\left(\boldsymbol{r}_{1}, \boldsymbol{r}_{2}\right)=a\left(r_{1}\right) b_{n}\left(r_{2}\right) \\
E_{n}^{(0)}=-2-1 /\left(2 n^{2}\right)
\end{gathered}
$$

where

$$
\begin{aligned}
a(r) & =(\pi / 8)^{-1 / 2} \exp (-2 r) \\
b_{n}(r) & =\left(n^{5} \pi\right)^{-1 / 2} \exp (-r / n) L_{n-1}^{1}(2 r / n)
\end{aligned}
$$

and $L_{n}^{m}$ is an associated Laguerre polynomial [18]. The wavefunction (4) can be antisymmetrized, as appropriate for a triplet state, but the zeroth-order energy (5) is unaffected by this.

\section{Hartree-Fock approximation}

The Fock operator [19] for the 1s ns triplet state is

$$
\boldsymbol{F}=-\frac{\nabla^{2}}{2}-\frac{2}{r}+\boldsymbol{J}_{1}-\boldsymbol{K}_{1}+\boldsymbol{J}_{n}-\boldsymbol{K}_{n}
$$

and iterative diagonalization of $\boldsymbol{F}$ in a complete basis, using the MOM [15] to guide convergence, yields the exact HF energy $E_{n}^{\mathrm{HF}}$.

We have used even-tempered Slater-type basis functions

$$
\chi_{k}(\boldsymbol{r})=\exp \left(-\alpha \beta^{k} r\right)
$$

with $\alpha=2$ and $\beta=160 / 173$ and $k=0,1, \ldots, 69$. Although such a basis cannot be complete [20], we have found empirically that it is sufficiently large to yield energies for the triplet $1 s n s$ states that are within a few nanohartrees of the HF limits for $n=$ $2,3, \ldots, 24$. The basis is almost linearly dependent and we have therefore performed the HF calculations using extended precision in the Mathematica package [21].

Suppose that we use the zeroth-order orbitals (6) and (7) as the initial guesses for an HF calculation. The extent to which they will change during the SCF procedure depends on the extent to which the potentials assumed in the zeroth-order Hamiltonian $H^{(0)}$ are incorrect. This is illustrated for the $n=10$ case in Figures 1 and 2.

The outer orbital $b_{10}(r)$ would remain unchanged if the inner electron shielded the nucleus perfectly

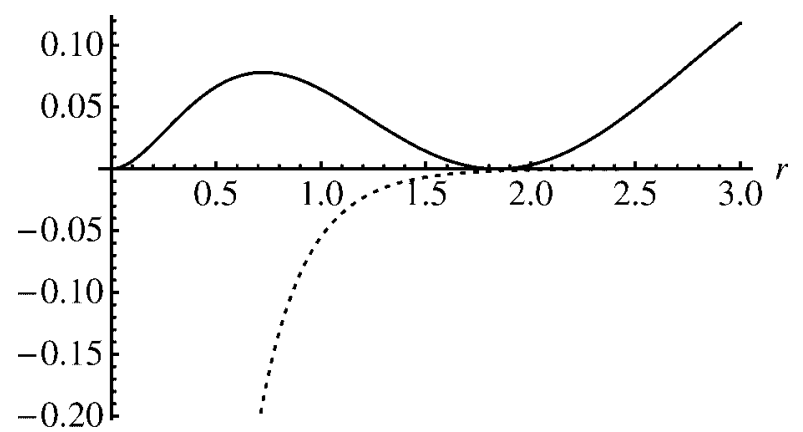

FIGURE 1. Radial density $(\times 200)$ of the outer orbital $b_{10}(r)$ (solid) and $V_{a}(r)-1 / r$ (dotted). 


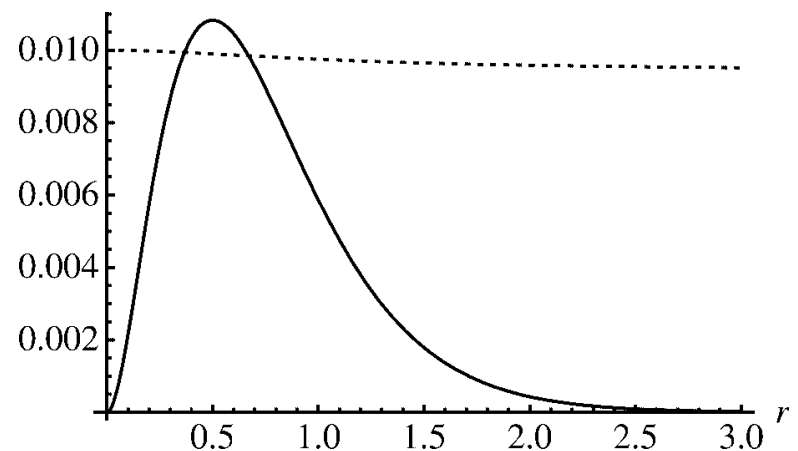

FIGURE 2. Radial density $(\times 0.01)$ of the inner orbital $a(r)$ (solid) and $V_{10}(r)$ (dotted).

or, in other words, if the Coulomb potential

$$
V_{a}(r)=\int \frac{a\left(\boldsymbol{r}^{\prime}\right)^{2}}{\left|\boldsymbol{r}-\boldsymbol{r}^{\prime}\right|} d \boldsymbol{r}^{\prime}
$$

of the inner orbital were exactly $1 / r$. However, as Figure 1 reveals, $V_{a}(r)$ significantly underestimates $1 / r$ in a region close to the nucleus where there is a small component of the outer orbital's density. As a result of this imperfect shielding, the outer orbital contracts towards the nucleus during the SCF, leading to a slight lowering of the system's energy.

The inner orbital $a(r)$ would remain unchanged if the outer electron had no effect on it or, in other words, if the Coulomb potential

$$
V_{n}(r)=\int \frac{b_{n}\left(\boldsymbol{r}^{\prime}\right)^{2}}{\left|\boldsymbol{r}-\boldsymbol{r}^{\prime}\right|} d \boldsymbol{r}^{\prime}
$$

of the outer orbital is constant in the region where the inner electron is found. By constructing the Taylor series of $V_{n}(r)$ around $r=0$, it can be shown that, near the nucleus, this potential is given by

$$
V_{n}(r)=\frac{1}{n^{2}}-\frac{2 r^{2}}{3 n^{3}}+\frac{2 r^{3}}{3 n^{3}}-\cdots
$$

and, as Figure 2 demonstrates, it is remarkably flat within the inner region. As a consequence, the inner orbital is essentially unaffected by the SCF and the optimized HF orbital remains extremely close to (6).

The inertness of the inner orbital greatly simplified our HF calculations because, by comparing our iterated energies with exact results for $n \leq 9$ from the Hartree-Fock Applet of Froese Fischer and Saparov [22], we found that its relaxation can be safely neglected for $n>5$. We could then find the outer orbital by performing a single diagonalization of the orthogonalized reduced Fock operator

$$
\boldsymbol{F}=-\frac{\nabla^{2}}{2}-\frac{2}{r}+J_{1}-K_{1}
$$

in our large Slater basis. This "frozen core" approach is, in fact, a special case of the Hartree-Fock Perturbation Theory (HFPT) that we have recently developed [17].

\section{Numerical results}

In Table I, we compare the zeroth-order energies from Eq. (5), our Hartree-Fock energies and the exact energies from the free ICI method [13] for the lowest triplet $1 \mathrm{~s} n \mathrm{~s}$ states of the helium atom. For convenience, we also give the resulting zeroth-order errors $\Delta_{n}^{(0)}=E_{n}^{(0)}-E_{n}^{\text {exact }}$ and correlation energies $E_{\text {corr }}=\Delta_{n}^{\mathrm{HF}}=E_{n}^{\mathrm{HF}}-E_{n}^{\text {exact }}$.

It is clear that the zeroth-order energies capture the essential behavior of the exact energies and that, as $n$ grows they become increasingly accurate. At $n=$ 24 , the error of the zeroth-order energy is only $22 \mu E_{\mathrm{h}}$ and the predicted ionization energy $\left(868 \mu E_{\mathrm{h}}\right)$ differs from the exact value $\left(890 \mu E_{\mathrm{h}}\right)$ by less than $3 \%$.

It is also clear that the HF energies follow the exact energies very faithfully and that $\Delta_{n}^{\mathrm{HF}}$ is typically almost two orders of magnitude smaller than $\Delta_{n}^{(0)}$. At $n=24$, the error of the HF energy (i.e., the correlation energy) is less than $0.3 \mu E_{\mathrm{h}}$ and the predicted ionization energy $\left(889.6 \mu E_{\mathrm{h}}\right)$ differs from the exact value $\left(889.9 \mu E_{\mathrm{h}}\right)$ by only $0.03 \%$. Evidently, if one is interested in such highly excited $1 s n s$ states, the simple Hartree-Fock approximation will be sufficiently accurate for many purposes.

Figure 3 shows $\log -\log$ plots of the variation of $\Delta_{n}^{(0)}$ and $\Delta_{n}^{\mathrm{HF}}$ with $n$, and reveals that both errors decay as $O\left(n^{-3}\right)$. As we have discussed earlier, the zeroth-order errors result primarily from the neglect of the relaxation of the outer orbital and we note, therefore, that first-order perturbation corrections will not give useful improvements. However, almost all of the error disappears at the HF level and the tiny remaining error (which is simply the electron correlation energy) can probably be understood by a Drude or London model.

\section{Concluding remarks}

As this work was nearing completion, we discovered an article by Kuriyan and Pritchard [23] 
DENG, GILBERT, AND GILL

TABLE I

Oth-order, HF, and exact energies of triplet $1 \mathrm{~s} n s$ states of He atom.

\begin{tabular}{|c|c|c|c|c|c|}
\hline$n$ & $-E_{n}^{(0)}$ & $-E_{n}^{\mathrm{HF}}$ & $-E_{n}^{\text {exact }}$ & $\Delta_{n}^{(0)}$ & $\Delta_{n}^{\mathrm{HF}}$ \\
\hline 2 & 2.12500000 & 2.17425078 & 2.17522938 & $5.0 \times 10^{-2}$ & $9.8 \times 10^{-4}$ \\
\hline 3 & 2.05555556 & 2.06848495 & 2.06868907 & $1.3 \times 10^{-2}$ & $2.0 \times 10^{-4}$ \\
\hline 4 & 2.03125000 & 2.03643642 & 2.03651208 & $5.3 \times 10^{-3}$ & $7.6 \times 10^{-5}$ \\
\hline 5 & 2.02000000 & 2.02258262 & 2.02261887 & $2.6 \times 10^{-3}$ & $3.6 \times 10^{-5}$ \\
\hline 6 & 2.01388889 & 2.01535734 & 2.01537745 & $1.5 \times 10^{-3}$ & $2.0 \times 10^{-5}$ \\
\hline 7 & 2.01020408 & 2.01111758 & 2.01112992 & $9.3 \times 10^{-4}$ & $1.2 \times 10^{-5}$ \\
\hline 8 & 2.00781250 & 2.00841901 & 2.00842712 & $6.1 \times 10^{-4}$ & $8.1 \times 10^{-6}$ \\
\hline 9 & 2.00617284 & 2.00659590 & 2.00660152 & $4.3 \times 10^{-4}$ & $5.6 \times 10^{-6}$ \\
\hline 10 & 2.00500000 & 2.00530675 & 2.00531079 & $3.1 \times 10^{-4}$ & $4.1 \times 10^{-6}$ \\
\hline 11 & 2.00413223 & 2.00436169 & 2.00436470 & $2.3 \times 10^{-4}$ & $3.0 \times 10^{-6}$ \\
\hline 12 & 2.00347222 & 2.00364833 & 2.00365063 & $1.8 \times 10^{-4}$ & $2.3 \times 10^{-6}$ \\
\hline 13 & 2.00295858 & 2.00309667 & 2.00309847 & $1.4 \times 10^{-4}$ & $1.8 \times 10^{-6}$ \\
\hline 14 & 2.00255102 & 2.00266130 & 2.00266273 & $1.1 \times 10^{-4}$ & $1.4 \times 10^{-6}$ \\
\hline 15 & 2.00222222 & 2.00231168 & 2.00231284 & $9.1 \times 10^{-5}$ & $1.2 \times 10^{-6}$ \\
\hline 16 & 2.00195313 & 2.00202669 & 2.00202764 & $7.5 \times 10^{-5}$ & $9.5 \times 10^{-7}$ \\
\hline 17 & 2.00173010 & 2.00179133 & 2.00179212 & $6.2 \times 10^{-5}$ & $7.9 \times 10^{-7}$ \\
\hline 18 & 2.00154321 & 2.00159471 & 2.00159538 & $5.2 \times 10^{-5}$ & $6.6 \times 10^{-7}$ \\
\hline 19 & 2.00138504 & 2.00142877 & 2.00142934 & $4.4 \times 10^{-5}$ & $5.6 \times 10^{-7}$ \\
\hline 20 & 2.00125000 & 2.00128745 & 2.00128793 & $3.8 \times 10^{-5}$ & $4.8 \times 10^{-7}$ \\
\hline 21 & 2.00113379 & 2.00116610 & 2.00116652 & $3.3 \times 10^{-5}$ & $4.1 \times 10^{-7}$ \\
\hline 22 & 2.00103306 & 2.00106114 & 2.00106150 & $2.8 \times 10^{-5}$ & $3.6 \times 10^{-7}$ \\
\hline 23 & 2.00094518 & 2.00096973 & 2.00097004 & $2.5 \times 10^{-5}$ & $3.1 \times 10^{-7}$ \\
\hline 24 & 2.00086806 & 2.00088965 & 2.00088992 & $2.2 \times 10^{-5}$ & $2.8 \times 10^{-7}$ \\
\hline
\end{tabular}

which includes accurate HF calculations of $1 \mathrm{~s} n \mathrm{~s}$ states of helium. Their energies agree well with ours for $n<20$ but, beyond this, there are growing discrepancies and their values imply that the correlation energy $\Delta_{n}^{\mathrm{HF}}$ begins to grow. Their HF energy for the $1 s 24 s$ state, for example, implies the correlation energy $\Delta_{24}^{\mathrm{HF}}=1.8 \mu E_{\mathrm{h}}$, which is almost an order of

$\log \Delta_{n}$

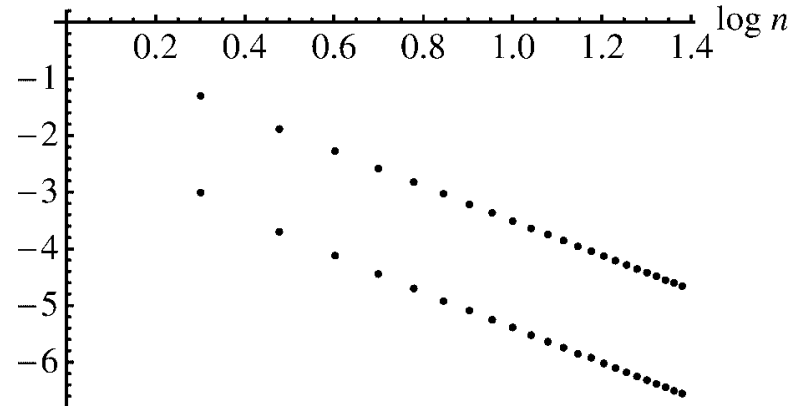

FIGURE 3. Variation of $\log \Delta_{n}^{(0)}$ (upper) and $\log \Delta_{n}^{\mathrm{HF}}$ (lower) with $\log n$. magnitude too large. For this reason, we believe that our energies are more accurate than theirs.

\section{References}

1. Iikura, H.; Tsuneda, T.; Yanai, T.; Hirao, K.; J Chem Phys 2001, 115,3540 .

2. Ewald, P. P. Ann Phys (Leipzig) 1921, 64, 253.

3. Savin, A. Recent Developments of Modern Density Functional Theory; Elsevier: Amsterdam, 1996.

4. Dombroski, J. P.; Taylor, S. W.; Gill, P. M. W. J Phys Chem 1996, $100,6272$.

5. Adamson, R. D.; Dombroski, J. P.; Gill, P. M. W. Chem Phys Lett 1996, 254, 329.

6. Gill, P. M. W.; Adamson, R. D. Chem Phys Lett 1996, 261, 105.

7. Toulouse, J.; Colonna, F.; Savin, A. Phys Rev A 2004, 70, 062505.

8. Gill, P. M. W.; Adamson, R. D.; Pople, J. A. Mol Phys 1996, 88, 1005.

9. Fock, V. Z Phys 1930, 61, 126.

10. Nakatsuji, H. J Chem Phys 2000, 113, 2949.

11. Nakatsuji, H. Phys Rev Lett 2004, 93, 030403.

12. Kurokawa, Y. I.; Nakashima, H.; Nakatsuji, H. Phys Chem Chem Phys 2008, 10, 4486. 
13. Nakashima, H.; Hijikata, Y.; Nakatsuji, H. J Chem Phys 2008, $128,154108$.

14. Bagus, P. S. Phys Rev 1965, 139, A619.

15. Gilbert, A. T. B.; Besley, N. A.; Gill, P. M. W. J Phys Chem A 2008, 112, 13164.

16. Besley, N. A.; Gilbert, A. T. B.; Gill, P. M. W. J Chem Phys (submitted).

17. Deng, J.; Gilbert, A. T. B.; Gill, P. M. W. J Chem Phys (submitted).
18. Abramowitz, M.; Stegun, I. E. Handbook of Mathematical Functions; Dover: New York, 1972.

19. Szabo, A.; Ostlund, N. S. Modern Quantum Chemistry; McGraw-Hill: New York, 1989.

20. Klahn, B.; Bingel, W. A. Theor Chim Acta 1977, 44, 27.

21. Wolfram Research, Inc., Mathematica, version 6.0 (2007).

22. Available at: http://atoms.vuse.vanderbilt.edu/hf.html.

23. Kuriyan, M.; Pritchard, H. O. Can J Chem 1976, 54, 1543. 